\title{
Simulation Monte Carlo du transport d'un panache plasma créé par ablation laser
}

\author{
F. Garrelie et A. Catherinot \\ Laboratoire SPCTS, UMR 6638 du CNRS, Équipe Plasma Laser Matériaux, \\ Faculté des Sciences, 123 avenue A. Thomas, 87060 Limoges, France
}

\begin{abstract}
Résumé : Le transport du panache plasma créé lors de l'interaction entre un laser à excimères et une cible de cuivre, à une fluence de $17 \mathrm{~J} / \mathrm{cm} 2$, est étudié par une simulation de type Monte Carlo. L'expansion sous vide du panache plasma, dans des conditions où la profondeur de matière évaporée excède largement quelques monocouches atomiques, est dominée d'une part par les processus collisionnels, liés à la forte densité de particules dans le panache de matière éjectée, et d'autre part, par la redistribution d'une partie de l'énergie incidente du faisceau laser sous forme d'énergie cinétique. L'influence d'une atmosphère résiduelle d'argon sur le transport des particules évaporées et sur le mélange des différentes particules est examinée. Une pression résiduelle d'argon supérieure à $50 \mathrm{~Pa}$ conduit à une compression des particules du gaz ambiant à l'avant du panache de matière éjectée, traduisant ainsi l'effet de chasse-meige du front du panache, observé expérimentalement.
\end{abstract}

\section{INTRODUCTION}

La réalisation de couches minces par photoablation d'une cible massive par un laser à excimères [1] suscite actuellement un grand intérêt dans de nombreux domaines. Afin de mieux comprendre les phénomènes de croissance de la couche mince, il apparaît nécessaire de contrôler le transport des espèces de la cible jusqu' au substrat. En raison du fort taux d'évaporation, les particules subissent suffisamment de collisions pour que les distributions finales des particules (près du substrat) soient très différentes des distributions initiales. Des études théoriques ont été proposées afin de décrire l'expansion de la bulle de matière éjectée [2-4] à partir des équations de la dynamique des fluides. Cependant, ces approches ne sont valables que lorsque la densité de particules est suffisamment élevée pour que le gaz puisse être considéré comme un fluide continu. Près du substrat, où s'effectue la croissance de la couche mince, la densité de matière éjectée est relativement faible, ne permettant pas de valider cette hypothèse. Jusqu'à maintenant, les simulations de type Monte Carlo ont été utilisées pour étudier l'effet des collisions entre les particules évaporées sous vide [5-7] à un taux d'évaporation relativement faible par rapport à celui obtenu par ablation laser, ou en présence d'une atmosphère résiduelle, par une méthode du type trajectoires aléatoires $[8,9]$, mais ne permettant pas une description du mouvement collectif des particules évaporées dans le gaz ambiant, ni du mouvement éventuel des particules du gaz ambiant, observés expérimentalement.

Dans cet article, nous présentons une simulation Monte Carlo du transport des espèces au-dessus de la cible lorsque ce transport s'effectue sous vide ou sous atmosphère résiduelle. Une simulation de l'expansion du panache dans des conditions similaires à celles rencontrées lors de la réalisation de couches minces par ablation par laser U.V. d'une cible massive a été développée. Les résultats mettent clairement en évidence le transfert d'énergie entre les particules évaporées de la cible et les particules du gaz ambiant.

\section{EXPANSION SOUS VIDE}

\subsection{Modèle}

L'expansion du panache plasma est simulée par une procédure Monte Carlo basée sur l'algorithme de Bird [10]. Compte tenu du nombre élevé de particules évaporées pendant la durée de l'impulsion laser, le 
panache est simulé en échelle réduite [11], autorisant ainsi la simulation avec un nombre réduit de particules.

La méthode proposée pour simuler le transport sous vide des particules a été présentée en détail par ailleurs [11]. Nous en rappellerons uniquement les principales caractéristiques. L'espace au-dessus de la cible est divisé en cellules repérées par deux coordonnées, $z$, normale à la surface de la cible et $r$, parallèle à la surface et mesurant la distance par rapport à l'axe $\mathrm{z}$. La simulation commence lorsque toutes les cellules du volume simulé sont vides. Pendant la durée de l'impulsion laser, les particules sont évaporées de la cible, en supposant un processus thermique d'évaporation. Le taux d'évaporation évolue à la fois dans le temps et en fonction de la position sur la surface de la cible [11]. Le transport et les interactions entre les particules sont calculés par intervalles de temps successifs, les collisions étant calculées à l'aide de la méthode du compteur de temps [10]. Afin de rendre compte de linfluence de l'absorption d'une partie de l'énergie incidente du faisceau laser par le panache de matière éjectée, nous supposons qu'une fraction des particules ionisées redistribue son énergie interne (énergie d'ionisation) sous forme d'énergie cinétique au cours de processus collisionnels de recombinaison [11].

\subsection{Résultats}

La courbe de temps de vol des particules à une hauteur de $5 \mathrm{~mm}$ au dessus de la cible, lorsque environ $6 \%$ de l'énergie incidente du faisceau laser est redistribuée sous forme d'énergie cinétique dans le panache plasma au cours de processus collisionnels de recombinaison, est reportée sur la figure 1. La courbe expérimentale, obtenue par spectroscopie optique d'émission (CuI, $\lambda=521.8 \mathrm{~nm}$ ) [11], y est également reportée.

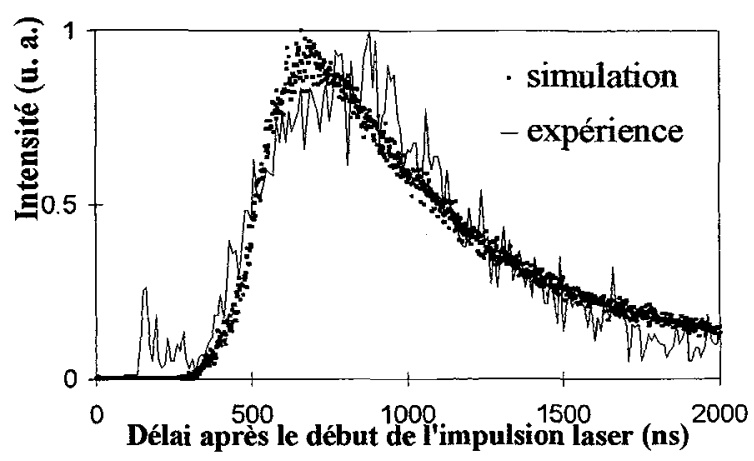

Figure 1 : courbes de temps de vol des particules à une hauteur de $5 \mathrm{~mm}$ au dessus de la surface de la cible lors de l'interaction entre un laser à excimères et une cible de cuivre, à une fluence de $17 \mathrm{~J} / \mathrm{cm}^{2}$.

Près de la surface de la cible (hauteurs inférieures à $4 \mathrm{~mm}$ ), seule la position temporelle du maximum de la courbe, directement liée à la vitesse la plus probable des particules, est correctement décrite par la simulation. En revanche, pour des distances supérieures à $4 \mathrm{~mm}$, la simulation reproduit correctement les distributions de vitesse des particules dans la direction normale à la surface de la cible, un bon accord est en effet observé à la fois sur la position temporelle du maximum de la courbe de temps de vol, et sur l'allure de ces courbes de temps de vol, ce qui tend à confirmer nos hypothèses.

\section{EXPANSION SOUS ATMOSPHERE RESIDUELLE}

\subsection{Modèle}

La méthode proposée pour étudier le transport sous vide des particules évaporées par ablation laser a été étendue au transport des particules sous une atmosphère résiduelle non réactive. Les particules évaporées 
de la cible et les particules du gaz environnant sont traitées de façon similaire, en dissociant le mouvement et les collisions sur des intervalles de temps successifs $\Delta t$. La phase d'évaporation des particules de cuivre est simulée de la même façon que précédemment (\$2.1). Les positions initiales des particules du gaz ambiant sont choisies aléatoirement dans le volume simulé. L'approche proposée ne distingue pas de phases temporelles au cours desquelles seules les interactions entre les particules évaporées ou seules les interactions entre les particules évaporées et les particules du gaz ambiant sont calculées. La probabilité de chaque type de collision est déterminée à partir de la section efficace de collisions et de la densité de particules de chaque espèce. Un test aléatoire basé sur ces différentes probabilités est alors utilisé pour déterminer le type de collision. Cette approche autorise l'étude du mouvement collectif des particules évaporées de la cible et des particules du gaz ambiant. Les différents types d'interaction entre ces particules peuvent intervenir au cours d'un même intervalle de temps, ce qui ne peut être effectué par un simulation du type trajectoires aléatoires $[8,9]$.

\subsection{Résultats}

Des simulations ont été effectuées pour différentes pressions résiduelles d'argon. Les distributions de densité des particules de cuivre et des particules d'argon, à un délai de $1 \mu$ s après le début de limpulsion laser et pour différentes pressions résiduelles d'argon sont reportées sur la figure 2 . Une augmentation de la pression résiduelle d'argon conduit à une compression des particules du gaz ambiant dans le front du panache de matière éjectée, en raison du transfert d'énergie cinétique au cours des collisions entre les particules du panache plasma et celles du gaz ambiant. Cet effet de "chasse-neige", observé expérimentalement $[12,13]$, résulte en un confinement du panache de matière éjectée et en une forte diminution de l'énergie cinétique des particules de cuivre. Une pression résiduelle d'argon supérieure à $50 \mathrm{~Pa}$ conduit à un mélange inhomogène des particules évaporées de la cible et des particules du gaz ambiant, une zone de forte densité de particules d'argon étant créée à la périphérie du panache, tandis que la zone centrale du plasma est déficitaire en particules du gaz ambiant.
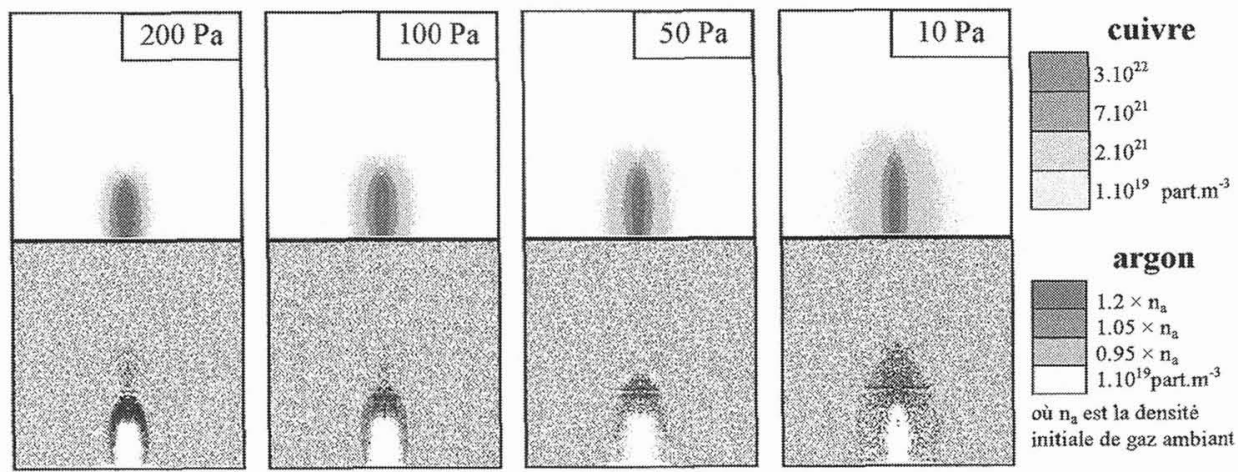

Figure 2 : distribution de la densité de particules de cuivre et d'argon à un délai de $1 \mu$ s après le début de l'impulsion laser, et pour différentes pressions résiduelles d'argon.

La figure 3 représente l'évolution temporelle de la hauteur atteinte par le front du panache de matière éjectée lors de l'expansion sous une pression résiduelle de $100 \mathrm{~Pa}$ d'argon. Cette évolution, sur l'intervalle de temps $0-1.2 \mu$ s, peut être décomposée en deux phases temporelles distinctes. Dans les premiers instants de l'expansion (jusqu'à environ $400 \mathrm{~ns}$ ), le panache s'étend librement au dessus de la cible, en raison de la différence de densité entre le panache plasma et le gaz environnant. En revanche, pour des délais plus élevés, l'évolution temporelle de la hauteur atteinte par le front du panache est assez bien décrite par l'équation de propagation d'une onde de souffle [14] $\left(\mathrm{h} \propto \mathrm{t}^{0.4}\right)$, en excellent accord avec les observations expérimentales $[12,13]$. La simulation permet donc de décrire les phénomènes hydrodynamiques majeurs régissant le transport du panache. 


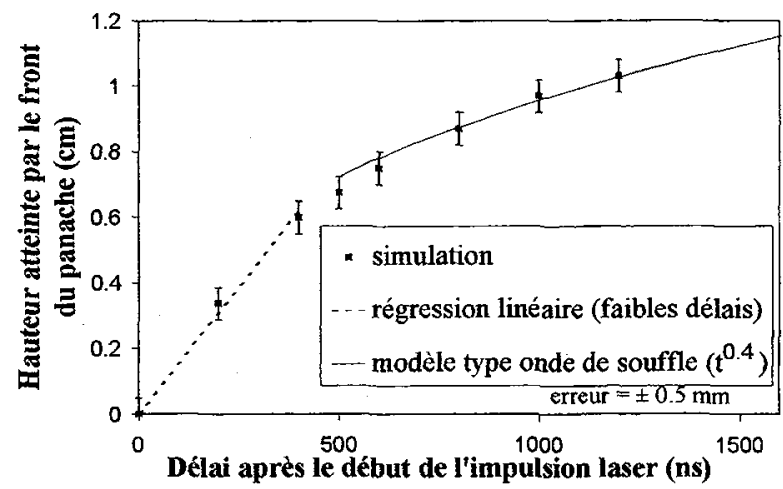

Figure 3 : simulation de l'évolution temporelle de la hauteur atteinte par le front du panache de matière éjectée, lors de l'expansion sous une pression résiduelle de $100 \mathrm{~Pa}$ d'argon.

\section{CONCLUSION}

Nous avons présenté une simulation, par une méthode Monte Carlo, de l'expansion du panache plasma créé lors de l'interaction entre un faisceau laser impulsionnel et une cible de cuivre.

Une méthode autorisant l'étude simultanée du transport des particules évaporées de la cible et des particules du gaz ambiant a été proposée. L'influence de différentes pressions résiduelles d'argon sur le transport des particules a été examinée. L'effet des collisions entre les particules évaporées de la cible et les particules du gaz ambiant est mis en évidence par la simulation. L'effet de chasse-neige du front du panache, observé expérimentalement, est en effet clairement observé par la simulation. Par ailleurs, l'évolution temporelle des dimensions du panache est en excellent accord avec les observations expérimentales. Une étude plus approfondie de linfluence, par exemple, de la nature et de la pression du gaz ambiant, peut maintenant être envisagée, avec d'évidentes applications à la réalisation de couches minces.

\section{Références}

[1] D.B. Chrisey and C.K. Hubler, Pulsed Laser Deposition of Thin Films (Naval Research Laboratory, Washington, DC 1994).

[2] R. K. Singh, O. W. Holland, J. Narayan, J. Appl. Phys 68(1), 233 (1990).

[3] J.C.S. Kools, T.S. Baller, S.T. de Zwart, J. Dieleman, J. Appl. Phys 71(9), 4547(1992).

[4] J. N. Leboeuf, K. R. Chen, J. M. Donato, D. B. Geohegan, C. L. Liu, A. A. Puretzky, Appl. Surf. Sci. 96-98, 14 (1996).

[5] I. Noorbatcha, R.R. Lucchese, Y. Zeiri, J. Chem. Phys. 86, 5816 (1987).

[6] D. Sibold, H.M. Urbassek, Phys Rev A 43, 6722 (1991).

[7] T. E. Itina, V. N. Tokarev, W. Marine, M. Autric, J. Chem. Phys. 106(21), 8905 (1997).

[8] J. C. S. Kools, J. Appl. Phys. 74(10), 6401 (1993).

[9] T. E. Itina, W. Marine, M. Autric, J. Appl. Phys. 82(7), 3536 (1997).

[10] G.A. Bird, Molecular Gas Dynamics (Clarendon, Oxford 1976).

[11] F. Garrelie, J. Aubreton, A. Catherinot, J. Appl. Phys. 83(10), 5075(1998).

[12] D. B. Geohegan, Appl. Phys. Lett. 60(22), 2732 (1992).

[13] B. Angleraud, C. Girault, C. Champeaux, F. Garrelie, C. Germain, A. Catherinot, Appl. Surf. Sci. 9698, 117(1996).

[14] Ya. B. Zel'dovich, Yu. P. Raizer, in Physics of Shock Waves and High Temperature Hydrodynamic Phenomena, Vol 1 (Academic Press, New York, 1966). 